\title{
20. Initial and Delayed Birefringence Signals and Membrane Potential of a Crayfish Giant Axon*)
}

\author{
By Akira Watanabe and Susumu Terakawa \\ Department of Physiology, Tokyo Medical and Dental University, \\ Yushima, Bunkyo-ku, Tokyo \\ (Comm. by Yasuji KAtsuki, M. J. A., Jan. 12, 1974)
}

In the previous report ${ }^{1)}$ we showed that the birefringence signal from a crab nerve is long-lasting and summates on repetitive stimulation. Furthermore, the signal is sensitive to drugs which are known to influence the intraaxonal structure. We therefore proposed that the birefringence signal is produced at least partly by structural changes within the axoplasm. However, since the crab nerve is a bundle of nerve fibers, an exact comparison with the membrane potential is hard to establish. In the present paper, we used the giant axon of a crayfish, in order to make a comparison between the optical signal and the intracellularly recorded action potential. It was found that the optical signal indeed summates on repetitive stimulation, in spite of the absence of corresponding summation of the action potential.

Methods. The median giant axon of a crayfish, at the level of the circumesophageal connective, was used as the experimental material. The nerve was desheathed and a part of the small fibers was removed with needles under a dissecting microscope, so that the median giant axon was clearly visible with the translucent light. Most axons we employed possessed diameters around $100 \mu$. The nerve was mounted horizontally in a chamber with two windows to pass the light beam. Stimulation was applied at the thoracic end of the axon, and the external action potential was recorded from the other end near the brain. The external medium was Harreveld's crayfish physiological saline unless otherwise noticed. The $\mathrm{pH}$ was adjusted to 7.2-7.5 by tris-HCl buffer or by bicarbonate buffer. Intracellular electrodes were of conventional type filled with $3 \mathrm{M}-\mathrm{KCl}$. The electrode was inserted into the axon at about the middle point of its illuminated stretch (about $4 \mathrm{~mm}$ ). The optical arrangements were similar to those described in the previous report. ${ }^{1)}$ With the use of a slit only the field occupied by the giant axon was illuminated. The optical signal was detected by a photodiode, amplified 3000 times and fed into Education.

*) This study was partly supported by a grant from the Ministry of 
a signal-averaging computor. Usually 1000 times accumulation was performed. The experiment was done in a room temperature which varied between $16-26^{\circ} \mathrm{C}$.

Results. For description of the birefringence signal, it is convenient to regard it as being composed of two phases. The initial response appears first and is often correlated with the shape of the action potential. The delayed response follows the initial response and can be recognized only by applying tetanic stimulation. It does not have any corresponding membrane potential change.

(A) The initial response. We subdivide the initial response into two components: the spike and the after-response. The spike appears first as a spike-like decrease of light intensity. The afterresponse follows the spike with a slower time course, and is a phase of either decreased or increased light intensity. The ratio of the two components was different according to preparations. A collection of the types of the initial response is presented in Fig. 1. Type $A$ is of triangular shape; no real spike-like phase is recognized. Type $B$ has a clear spike which is followed by an after-response with the same direction as the spike (i.e. the direction of decrease in light intensity). Type $C$ is characterized by a lack of after-response; the spike directly returns to the base line. Type $D$ has an after-response with the direction reversed to that of the spike (i.e. the direction of increase in light intensity). The above classification is employed simply for convenience of description; intermediate forms exist which have to be classified into one of the types more or less arbitrarily. During the course of the present experiment we examined 55 preparations. The rates of appearance of the types were; Type A, 25\%; Type B, $31 \%$; Type C, $20 \%$; Type D, $24 \%$.
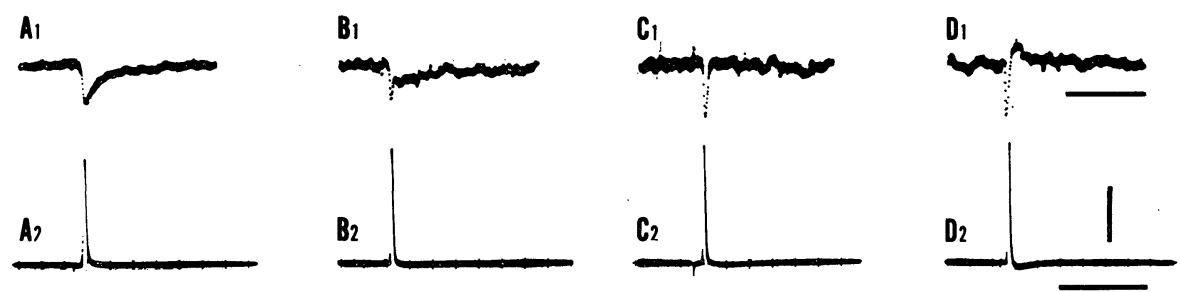

Fig. 1. Birefringence signals (upper traces) and intracellularly recorded action potentials (lower traces) from four different axons. In all optical records in this paper the downward deflection indicates decrease in light intensity. $A_{1}, B_{1}, C_{1}$ and $D_{1}$ are examples of Type A, Type B, Type C and Type D optical signals, respectively. The approximate spike amplitudes of the optical signals (per impulse, divided by background light intensity) were: $A_{1}: 5.8 \times 10^{-5}, B_{1}: 2.2 \times 10^{-5}, C_{1}: 1.3 \times 10^{-5}, D_{1}: 1.2 \times 10^{-5}$. The horizontal bars indicate $50 \mathrm{msec}$. The vertical bar represents $50 \mathrm{mV} .16-17^{\circ} \mathrm{C}$. 
Correlation with the action potential. Cohen et al. ${ }^{2)}$ found that the birefringence signal from the squid giant axon closely follows the time course of the membrane potential. In the crayfish giant axon, however, the optical signal did not always follow the time course of the membrane potential. The discrepancy was largest in Type A optical signals, where the spike was absent. However, in Types B, $\mathrm{C}$ and $\mathrm{D}$, we recognized some resemblance to the membrane potential change, because the optical response had an initial spike with its shape and duration similar to those of the electrical spike potential. Furthermore, there was a close correlation between the types of optical responses and the direction of the after-potential. Thus, when the action potential had an after-depolarization, most axons produced optical signals of Type A or Type B; whereas when the action potential had an after-hyperpolarization, most axons produced optical signals of Type D. Some other pieces of evidence also indicated the existence of the correlation. (1) With replacement of the external medium from the standard Harreveld solution to the one with reduced univalent cation concentrations and an increased Ca concentration, it was possible to change an after-hyperpolarization to an after-depolarization. ${ }^{3}$ Associated with this change, the optical signal changed from Type D to Type B. (2) Application of hyperpolarizing current to the membrane with the aid of a second intracellular electrode changed an after-hyperpolarization to an after-depolarization. Correspondingly, the optical signal changed from Type D to Type A or Type B.

However, we also found several cases where the action potential had an after-depolarization but the optical signal was of Type D, or the action potential had an after-hyperpolarization but the optical signal was of Type A or of Type B (see, Fig. 1B). Therefore the matching of the directions of optical after-response and electrical after-potential was not a rigid rule. We also observed the following phenomenon consistently. Originally an axon showed an afterhyperpolarization and an optical response of Type D. On application of repetitive stimulation, the after-potential changed its direction and became an after-depolarization. In spite of this, the optical signal remained to be the same as before (see, Fig. $2 B$ ).

(B) The slow optical signals in response to repetitive stimulation. When the axon was repetitively stimulated at a frequency around $100 \mathrm{~Hz}$, the optical signal usually summated, and after stimulation a conspicuous tail of the signal was observed in most preparations. In Fig. $2 A$, a single stimulus elicited an initial optical response of Type A. On repetitive stimulation, the base line of spike responses lowered gradually, apparently because of the summation of after- 
$A_{1}$
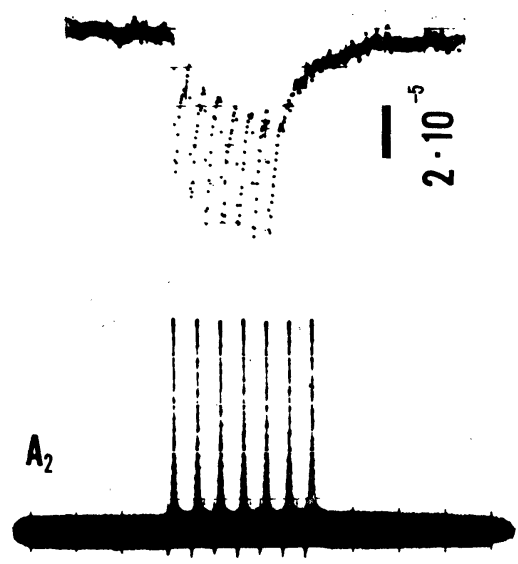

$B_{1}$

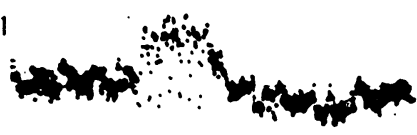

$100 \mathrm{msec}$

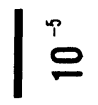

$111 ! 11 !$

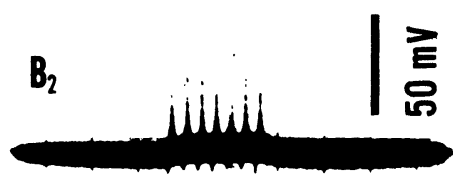

$100 \mathrm{msec}$

Fig. 2. Summation of optical signals in response to tetanic stimulation (upper traces) and simultaneously recorded action potentials (lower traces). Trains of 7 shocks were applied repetitively with a period of 0.8 sec. to accumulate the optical signal. $A_{1}$ and $A_{2}$ : from the same axon as that shown in Fig. 1A. $B_{1}$ and $B_{2}$ : from another axon which produced a Type $\mathrm{D}$ optical response and an action potential with an after-hyperpolarization in response to a single stimulus. During the initial stage of repetitive stimulation the afterpotentials were changed gradually to become after-depolarizations, as shown in $B_{2} .22^{\circ} \mathrm{C}$.

responses. The summation of the after-potential was of the order of $0.2 \mathrm{mV}$. After the period of repetitive stimulation, the lowered base line of the optical signal subsided gradually with a time constant of approximately $50 \mathrm{msec}$. In Fig. $2 B$, a single stimulus elicited an initial optical response of Type D. The summation of after-responses produced a slight elevation of the base line. However, in a brief period the base line turned the direction and started falling. After the period of stimulation, the lowered base line was remained and gradually subsided. A comparison of the above two examples reveals that there are two kinds of signals which constitute the slow phase of the optical response. One of them is the summated after-response, which takes the same direction as that of the after-response. Another is an optical signal which develops more gradually and which always takes the direction of decrease in light intensity, irrespective of the direction of the after-response. We call this slower response the delayed response. As seen in Fig. 2, it was impossible to observe any membrane potential change associated with the delayed response.

Experiments with a quater-wave plate. In experiments shown 

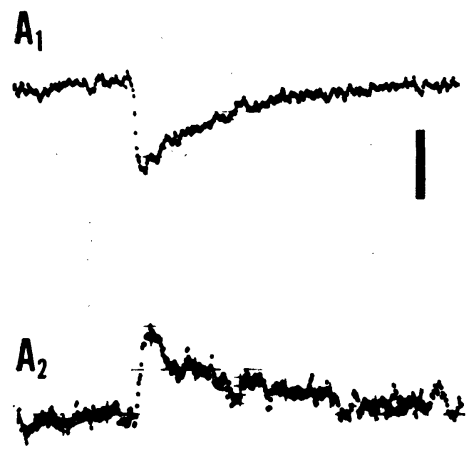

$20 \mathrm{msec}$
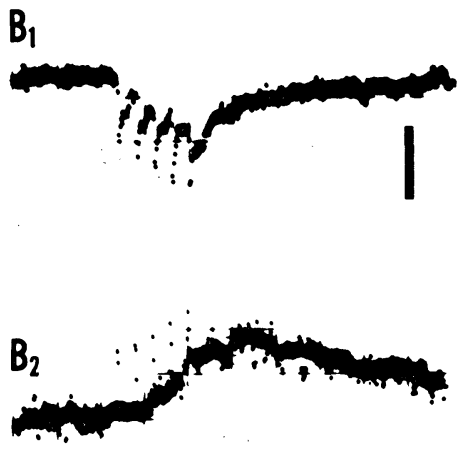

$100 \mathrm{msec}$

Fig. 3. Reversal of the optical signal with a quater-wave plate. $A_{1}$ and $B_{1}$ : original signals recorded under the cross-polar condition. The vertical bars indicate $10^{-5}$ of the background light intensity. $A_{2}$ and $B_{2}$ : reversed signals by insertion of the quater-wave plate. In $A_{2}$ the gain of the signal recording was one half of that in $A_{1}$, and in $B_{2}$ it was one fourth of that in $B_{1}$. The background light.intensity was increased by insertion of the quaterwave plate. $A$, a response to a single stimulus of an axon. Ca concentration of the external medium was tripled in this particular experiment. $B$, a response to tetanic stimulation of another axon in the standard Harreveld saline. $22^{\circ} \mathrm{C}$.

in Fig. 3, the optical signal was reversed by inserting a quater-wave plate between the preparation and the analyzer. The time course of the reversed signal was different from that of the original signal, indicating that factors other than the birefringence change contribute to produce the optical signal recorded under the usual cross-polar conditions. Nevertheless, the reversal indicates that the birefringence change is a cause of the slow optical signal.

Discussion. The results show that the electrical and optical signals match only to a limited extent. The shape of the initial optical response was sometimes similar to that of the action potential, but more often the optical response showed a prominent and long-lasting after-response, in spite of the fact that the electrical after-potential was always very small in this axon. The directions of the optical afterresponse and the electrical : after-potential usually matched but even this rule was not universal but allowed exceptions. The time course of the Type A optical signal differed widely from that of the membrane potential. The summation of the optical after-response was distinct, but the summation of the electrical after-potential was very small or absent. The delayed optical response took place without any recognizable membrane potential change. We thus conclude that in addition to the effect of the membrane potential the change in birefringence of the axon is produced by some other structural 
changes of the axon. The experimental results in the present paper do not supply any direct information concerning the nature of the other changes which produce the signal. However, based on an analogy with the case of the crab nerve, ${ }^{1)}$ we suspect that a considerable part of the signal might originate from structural changes taking place within the axoplasm.

\section{References}

1) Watanabe, A., Terakawa, S., and Nagano, M. (1973) : Proc. Japan Acad., 49, 470.

2) Cohen, L. B., Hille, B., and Keynes, R. D. (1970): J. Physiol., 211, 495.

3) Yamagishi, S., and Grundfest, H. (1971): J. Membrane Biol., 5, 345. 